\title{
Effective Action and Field Equation for BEC from Weak to Strong Couplings
}

\author{
Hagen Kleinert* \\ Institut für Theoretische Physik, Freie Universität Berlin, 14195 Berlin, Germany and \\ ICRANeT Piazzale della Repubblica, 10 -65122, Pescara, Italy
}

\begin{abstract}
While free and weakly interacting nonrelativistic particles are described by a Gross-Pitaevskii equation, which is a nonlinear self-interacting Schrödinger equation, the phenomena in the strongcoupling limit are governed by an effective action that is extremized by a double-fractional generalization of this equation. Its particle orbits perform Lévy walks rather than Gaussian random walks.
\end{abstract}

PACS numbers: 95.35+d.04.60,04.20C,04.90

\section{INTRODUCTION}

A quarter of a century after Bose and Einstein's 19241925 prediction [1, 2] that a free gas of Bose particles would condense at low temperature to a coherent state, Bogoliubov developed a first theory of weakly interacting Bose-Einstein condensates (BEC) in 1947 [3]. It was an inspiring paper for many similar condensation phenomena, such as superfluidity and superconductivity. The experimental study of a BEC had to wait until 1995. Only then were cooling and trapping techniques sufficiently well developed to prepare large enough samples of such gases to observe their physical properties. Presently these supply us with important systems on which we can test the theoretical tools developed in many-body physics and quantum field theory.

As an important result it was found that a nonlinear Schrödinger equation, which in this context is known as Gross-Pitaevskii equation, yields satisfactory descriptions of such condensed states if the temperature is sufficiently low [4, 5]. This is possible due to the high dilution of the condensates which ensures the weakness of the interaction of the particles.

More recently, however, it has been possible to increase the interaction strength of these gases so much that in spite of the high dilution, the interactions can be considered as strong. The way to do is to exploit the so-called Feshbach resonance in the two-body potential. There exists a magnetic field $B_{c}$ where the scattering length of the two-body potential grows to infinity [7]. In that regime, the Bogoliubov theory is no longer applicable, and the quantum field theory of the Bose gas becomes completely nontrivial and therefore interesting.

If we want to carry quantum field theoretical calculations from the well understood weak-coupling regime to strong couplings, we may resort to various procedures. One is, of course, the numerical simulation of the partition function of the system on a computer. This may yield numerical date with can be compared with experiments. A more satisfactory understanding can, however, be reached by analytic methods. If the action of the systems is formulated on a lattice, there are high- temperature and low-temperature expansions. The critical regime between them remains, however, hard to reach since both expansions diverge where they are supposed to meet. The problem is that one needs in principle infinitely many orders to reach a critical point.

In the past twenty years, methods have been developed to use weak-coupling expansions and resumming them in the strong-coupling regime. This is known as the renormalization group approach [8]. If the field theory is formulated in the continuum, the weak-coupling expansions require the calculation of Feynman diagrams. The critical point lies at an infinite coherence length which corresponds to a zero mass of the euclidean field theory. At that point, many Feynman diagrams diverge. If we want to study the limiting theory, we must renormalize the theory. For this we introduce an arbitrary mass scale $\mu$, and for each $\mu$ we calculate a renormalized coupling strength $g$. The resulting expansions in powers of $g$ are all divergent with a vanishing radius of convergence. They need sophisticated mathematical methods of resummation.

There exists a a renormalization group function $\beta(g)$ which controls the change of the renormalized coupling constant under a change of the renormalization scale $\mu$ 9, 10]. If this function has a fixed point $g^{*}$ in the infrared limit $\mu \rightarrow 0$, then the resummed series yield reasonable estimates for the critical behavior of a theory. The general form of the physical laws in this limit have been formulated by many authors 11,13 .

A much simpler and yet most powerful method has been developed in the textbook [14]. Again, the basic input consists in the diagrammatic expansions of the amplitudes, but here expressed directly in powers of the un renormalized or bare coupling constant $g_{B}$. These expansions are subjected to a variational procedure [15. that converts the divergent weak-coupling power series into convergent strong-coupling power series [16]. Their expansion parameter is a certain inverse power of the coupling constant, $1 / g_{B}^{\omega}$. The parameter $\omega$ is the socalled exponent governing the approach to scaling, introduced by Wegner in 1990 [17]. The new method is called Variational Perturbation Theory (VPT). If applied to quantum mechanics it reproduces the same results as the old-fashioned $\delta$-expansion [18]. In quantum field the- 
ory, however, there are anomalous power laws, which cannot be handled by the $\delta$-expansion, and the full VPT is essential. It has so far given the most accurate critical exponents measured by precision experiments in microgravity satellite environment [19].

For various physical quantities and critical exponents, the critical expressions and the approach to the critical limit have been given in the textbook [14]. It is the purpose of this paper to extend the method to the calculation of effective actions. From perturbation theory it is well known how to calculate the effective action as a power series in the field strength, to be called $\Phi$. This expansion is valid as long as $\Phi$ remains small. For large field strength, however, the effective action exhibits a nontrivial power behavior which is approached in a way controlled again by the Wegner exponent $\omega$. We shall present a method for finding a global expression the effective action as a function of of any $\Phi$. It has for small $\Phi$ the correct power series, and for large $\Phi$ the correct power behavior, with the proper approach to the scaling limit.

\section{GENERATING FUNCTIONAL AND EFFECTIVE ACTION}

The physical properties of a system described by a scalar quantum field $\phi(x)$ can be derived from a classical effective action that is derived as follows. One starts from the generating functional of all Green functions

$$
Z[j]=e^{i W[j] / \hbar},
$$

where $W[j]$ is the generating functional of all connected Green functions. The vacuum expectation of the field, the average

$$
\Phi(x) \equiv\langle\phi(x)\rangle,
$$

is given by the first functional derivative

$$
\Phi(x)=\delta W[j] / \delta j(x) .
$$

This can be inverted to yield $j(x)$ as an $x$-dependent functional of $\Phi(x)$ :

$$
j(x)=j[\Phi](x) .
$$

This is used to form the Legendre transform of $W[j]$ :

$$
\Gamma[\Phi] \equiv W[j]-\int d^{4} x j(x) \Phi(x) .
$$

where $j(x)$ on the right-hand side is replaced by (44). This is the effective action of the theory. Its first functional derivative gives back the current

$$
\frac{\delta \Gamma[\Phi]}{\delta \Phi(x)}=-j(x) .
$$

In the absence of an external current $j(x)$, the effective action is extremal on the physical field expectations $\Phi(x)$.

The generating functional of all connected Green functions can be recovered from the effective action by the inverse Legendre transform

$$
W[j]=\Gamma[\Phi]+\int d^{4} x j(x) \Phi(x) .
$$

In general, the above quantities can be obtained with the help of functional integrals. The generating functional $Z[j]$, for example, is given by

$$
Z[j]=\frac{\int \mathcal{D} \phi(x) e^{(i / \hbar)\left\{\mathcal{A}[\phi]+\int d^{4} x j(x) \phi(x)\right\}}}{\int \mathcal{D} \phi(x) e^{(i / \hbar) \mathcal{A}_{0}[\phi]}} .
$$

Using (11) and (5), this amounts to the functional integral formula for $\Gamma[\Phi]$ :

$$
\begin{aligned}
& e^{\frac{i}{\hbar}\left\{\Gamma[\Phi]+\int d^{4} x j(x) \Phi(x)\right\}} \\
& \quad=\mathcal{N} \int \mathcal{D} \phi(x) e^{(i / \hbar)\left\{\mathcal{A}[\phi]+\int d^{4} x j(x) \phi(x)\right\}}
\end{aligned}
$$

with the normalization factor

$$
\mathcal{N}=\left\{\int \mathcal{D} \phi(x) e^{(i / \hbar) \mathcal{A}_{0}[\phi]}\right\}^{-1} .
$$

In writing this we have explicitly displayed the fundamental action quantum $\hbar$, which is a measure for the size of quantum fluctuations. There most physical systems, the quantum fluctuations are rather small except in the immediate vicinity of critical points.

For $\hbar \rightarrow 0$, the path integral over the field $\phi(x)$ in (88) is dominated by the classical solution $\phi_{\mathrm{cl}}(x)$ which extremizes the exponent

$$
\left.\frac{\delta \mathcal{A}}{\delta \phi}\right|_{\phi=\phi_{\mathrm{cl}}(x)}=-j(x) .
$$

At this level we therefore identify

$$
\begin{aligned}
W[j] & =\Gamma[\Phi]+\int d^{4} x j(x) \Phi(x) \\
& =\mathcal{A}\left[\phi_{\mathrm{cl}}\right]+\int d^{4} x j(x) \phi_{\mathrm{cl}}(x) .
\end{aligned}
$$

Of course, $\phi_{\mathrm{cl}}(x)$ is a functional of $j(x)$, so that we may write it more explicitly, as $\phi_{\mathrm{cl}}[j](x)$. By differentiating $W[j]$ with respect to $j$, we have from the general first part of Eq. (2):

$$
\Phi(x)=\frac{\delta W}{\delta j}=\frac{\delta \Gamma}{\delta \Phi} \frac{\delta \Phi}{\delta j}+\Phi+j \frac{\delta \Phi}{\delta j} .
$$

Inserting the classical field equation (11), this becomes

$$
\Phi(x)=\frac{\delta \mathcal{A}}{\delta \phi_{\mathrm{cl}}} \frac{\delta \phi_{\mathrm{cl}}}{\delta j}+\phi_{\mathrm{cl}}+j \frac{\delta \phi_{\mathrm{cl}}}{\delta j}=\phi_{\mathrm{cl}}
$$


Thus, to this approximation, $\Phi(x)$ coincides with the classical field $\phi_{\mathrm{cl}}(x)$. Replacing $\phi_{\mathrm{cl}}(x) \rightarrow \Phi(x)$ on the right-hand side of Eq. (12), we therefore obtain the lowest-order result (zeroth order in $\hbar$ ):

$$
\Gamma[\Phi]=\mathcal{A}[\Phi]
$$

i.e., the effective action equals the fundamental action.

In general it can be shown that the effective action can be expanded in a functional power series of $\Phi(x)$

$$
\begin{aligned}
\Gamma[\Phi] & =\sum_{n=0}^{\infty} \frac{1}{n !} \int d^{D} x_{1} d^{D} x_{2} \cdots d^{D} x_{n} \Gamma^{(n)}\left(x_{1}, x_{2}, \ldots, x_{n}\right) \\
& \times \Phi\left(x_{1}\right) \Phi\left(x_{2}\right) \cdots \Phi\left(x_{n}\right) .
\end{aligned}
$$

where the $n$th coefficient is just the $n$-point vertex function of the theory $[\underline{6}, 14]$.

For the $\phi^{4}$-theory with $\mathrm{O}(N)$-symmetry and action

$$
\mathcal{A}[\phi]=\int d^{4} x\left[\frac{1}{2}\left(\partial \phi_{a}\right)^{2}-\frac{m^{2}}{2} \phi_{a}^{2}-\frac{g}{4 !}\left(\phi_{a}^{2}\right)^{2}\right],
$$

the zeroth-order effective action is

$$
\Gamma[\Phi]=\int d^{4} x\left[\frac{1}{2}\left(\partial \Phi_{a}\right)^{2}-\frac{m^{2}}{2} \Phi_{a}^{2}-\frac{g}{4 !}\left(\Phi_{a}^{2}\right)^{2}\right] .
$$

For $m^{2}>0,{ }^{1}$ this has an extremum at $\Phi_{a} \equiv 0$, and there are only two non-vanishing vertex functions $\Gamma^{(n)}\left(x_{1}, \ldots, x_{n}\right)$ :

$\boldsymbol{n}=2$ :

$$
\begin{aligned}
\Gamma_{a b}^{(2)}\left(x_{1}, x_{2}\right) & \left.\equiv \frac{\delta^{2} \Gamma}{\delta \Phi_{a}\left(x_{1}\right) \delta \Phi_{b}\left(x_{2}\right)}\right|_{\Phi_{a}=0} \\
& =\left.\frac{\delta^{2} \mathcal{A}}{\phi_{a}\left(x_{1}\right) \phi_{b}\left(x_{2}\right)}\right|_{\phi_{a}=\Phi_{a}=0} \\
& =\left(-\partial^{2}-m^{2}\right) \delta_{a b} \delta^{(4)}\left(x_{1}-x_{2}\right) .
\end{aligned}
$$

This determines the inverse of the propagator:

$$
\Gamma_{a b}^{(2)}\left(x_{1}, x_{2}\right)=\left[i \hbar G^{-1}\right]_{a b}\left(x_{1}, x_{2}\right),
$$

Thus we find to this zeroth-order approximation that $G_{a b}\left(x_{1}, x_{2}\right)$ is equal to the free propagator:

$$
G_{a b}\left(x_{1}, x_{2}\right)=G_{0 a b}\left(x_{1}, x_{2}\right)
$$

$$
\begin{aligned}
& n=4: \\
& \begin{aligned}
\Gamma_{a b c d}^{(4)}\left(x_{1}, x_{2}, x_{3}, x_{4}\right) & \equiv \frac{\delta^{4} \Gamma}{\delta \Phi_{a}\left(x_{1}\right) \delta \Phi_{b}\left(x_{2}\right) \delta \Phi_{c}\left(x_{3}\right) \delta \Phi_{d}\left(x_{4}\right)} \\
& =g T_{a b c d},
\end{aligned}
\end{aligned}
$$

\footnotetext{
1 The case $m^{2}<0$ corresponds to the condensed phase of the system and will be treated below.
}

with

$$
T_{a b c d}=\frac{1}{3}\left(\delta_{a b} \delta_{c d}+\delta_{a c} \delta_{b d}+\delta_{a d} \delta_{b c}\right),
$$

which is just the fundamental vertex implied by the local interaction (18).

According to the definition of the effective action, all diagrams of the theory can be composed from the propagator $G_{a b}\left(x_{1}, x_{2}\right)$ and this vertex via tree diagrams. Thus we see that in this lowest approximation, we recover precisely the subset of all original Feynman diagrams with a tree-like topology. These are all diagrams which do not involve any loop integration. Since the limit $\hbar \rightarrow 0$ corresponds to the classical equations of motion with no quantum fluctuations we conclude: Classical field theory corresponds to quantum field theory in the tree approximation.

The use of the initial action as an approximation to the effective action neglecting fluctuations is often referred to as mean-field theory.

Let us now apply the effective action formalism to the phenomenon of Bose-Einstein condensation (BEC). There the number of $\mathrm{O}(N)$ components is $N=2$, and $\Phi^{2}$ is identified with $\Psi^{\dagger} \Psi / M k_{B} T$, and the interaction with $\left(g_{S} / 2\right) \int d^{D}\left(\Psi^{\dagger} \Psi\right)^{2}$, the subscript $S$ indicating that the lowest-order equation of motion of the field $\Phi$ becomes the nonlinear Schrödinger equation

$$
\left[-i \hbar \partial_{t}-\frac{\hbar^{2}}{2 M} \nabla^{2}-\mu+g_{S} \Psi^{\dagger} \Psi\right] \Psi(x)=0,
$$

where $M$ is the physical mass of the particles. The relation with the previous coupling constant is

$$
g_{S} / 2=g / 4 ! .
$$

Here $\mu$ is the chemical potential of the particles which is fixed by ensuring a given particle number $N$.

After quantization, the nonlinear Schrödinger equation (143) has a phase transition at a temperature $T_{c}$ where the chemical potential $\mu$ vanishes. For a free gas, this is determined by the equation

$$
N=\sum_{\mathbf{p}} \frac{1}{e^{\beta \hbar \omega_{\mathbf{p}}-\beta \mu}-1},
$$

where $\beta=1 / k_{B} T, k_{B}$ is the Boltzmann constant, and $\omega_{\mathbf{p}}=\mathbf{p}^{2} / 2 M$ the kinetic energy of the particles. In $D$ dimensions, (26) can be written as an energy integral

$$
N=\frac{1}{\Gamma(D / 2)} \frac{V_{D}}{\sqrt{2 \pi \hbar^{2} \beta / M}} \int_{0}^{\infty} d \varepsilon \frac{\varepsilon^{D / 2-1}}{e^{\varepsilon-\beta \mu}-1}
$$

where $V_{D}=$ volume. For $\mu=0$, the integral yields Riemann's zeta function $\zeta(D / 2)=\sum_{n=1}^{\infty} n^{-D / 2}$, and we find the critical temperature from

$$
N=\frac{1}{\Gamma(D / 2)} \frac{V_{D}}{{\sqrt{2 \pi \hbar^{2} \beta_{c} / M}}^{D}} \zeta(D / 2),
$$


In the neighborhood of $T_{c}$ and for $D=3$, the chemical potential behaves like

$$
-\mu \approx \frac{1}{4 \pi} k_{B} T_{c} \zeta^{2}(3 / 2)\left[\left(\frac{T}{T_{c}}\right)^{3 / 2}-1\right]^{2} .
$$

This is the critical regime of the Bose gas. Its statistical properties are governed by the action

$$
\mathcal{A}=\beta \int d^{D} x\left[-\frac{\hbar^{2}}{2 M} \partial \Psi^{*} \partial \Psi+\mu \Psi^{*} \Psi-\frac{g_{S}}{2}\left(\Psi^{*} \Psi\right)^{2}\right]
$$

The coupling constant $g_{S}$ can be expressed in terms of the $s$-wave scattering length $a_{s}$ of the atoms as $M g_{S}=$ $4 \pi \hbar^{2} a_{s}$ [5]. The connection with the action (17) is established by equating $m^{2}=-2 M \mu$ and $g=4 ! 2 \pi a_{s} M k_{B} T$ which has near $T_{c}$ is, in natural units with $\hbar=1$, $c=1$, the approximate size $g \approx 4 ! 2 \pi a_{s} / \ell_{c}^{2}$ where $\ell_{c}=\left[\zeta(D / 2) V_{D} / N \Gamma(D / 2)\right]^{1 / D}$.

If the BEC takes place in an external trap potential $V(\mathbf{x})=M \omega^{2} \mathbf{x}^{2} / 2$, the action (30) contains, instead of the chemical potential $\mu$, an $\mathbf{x}$-dependent chemical potential $\mu(\mathbf{x})=\mu+M \omega^{2} \mathbf{x}^{2} / 2$, and the particle density $n(\mathbf{x})=\Psi^{*} \Psi$ is obtained from the solution of the effective field equation

$$
\left[-\frac{\hbar^{2}}{2 M} \nabla^{2}+\mu+M \omega^{2} \mathbf{x}^{2} / 2+g_{S} n(\mathbf{x})\right] \Psi(x)=0 .
$$

In the Thomas-Fermi approximation, we neglect the gradient term and find the density profile

$$
n(\mathbf{x})=\frac{M \omega^{2}}{g_{S}}\left(R_{c}^{2}-R^{2}\right), \quad R^{2}=\mathbf{x}^{2},
$$

where $M \omega^{2} R_{c}^{2} \equiv-\mu$.

The interaction shifts the critical temperature of the BEC in $D=3$ dimensions from the free-particle value determined by (28) to a higher value $T_{c}+\Delta T_{c}$ with $\Delta T_{c} / T_{c} \approx a_{s}\left(N / V_{3}\right)^{1 / 3}[21]$.

The reason for the good agreement with observations is the smallness of the coupling constant $g_{S}$ which, as we discussed before, is ensured in experimental BEC by the diluteness of the atomic gases that can be brought to the low critical temperatures in the laboratory. If the coupling gets larger, the interaction changes the properties of the gas, and the extremization of the action (30) will no longer be sufficient to explain the physical properties of the gas. Instead, one has to extremize the effective action $\Gamma[\Phi]$, to which (30) is merely the mean-field approximation.

An important experimental method to investigate the strong-coupling properties of the gas is based on placing the sample in a magnetic field $B$ and raising this up to a critical value $B_{c}$, where the two-particle scattering length diverges at a so-called Feshbach resonance. At that point one reaches the strong-coupling limit of the the field theory (30). The purpose of this note is to calculate the full effective action $\Gamma[\Phi]$ of the theory for all couplings, from weak to strong.

\section{QUADRATIC FLUCTUATIONS}

Let us calculate the higher $\hbar$-correction to the meanfield approximation (15). For this we expand the action in (9) in powers of the fluctuations of the field around the classical solution

$$
\delta \phi(x) \equiv \phi(x)-\phi_{\mathrm{cl}}(x)
$$

and perform a perturbation expansion. The quadratic term in $\delta \phi(x)$ is considered as a "free-field action", the higher powers in $\delta \phi(x)$ as "interactions". Up to second order in the fluctuations $\delta \phi(x)$, the action is expanded as follows:

$$
\begin{aligned}
\mathcal{A}\left[\phi_{\mathrm{cl}}+\delta \phi\right] & +\int d^{4} x j(x)\left[\phi_{\mathrm{cl}}(x)+\delta \phi(x)\right] \\
=\mathcal{A}\left[\phi_{\mathrm{cl}}\right]+ & \int d^{4} x j(x) \phi_{\mathrm{cl}}(x) \\
+ & \int d^{4} x\left\{j(x)+\left.\frac{\delta \mathcal{A}}{\delta \phi(x)}\right|_{\phi=\phi_{\mathrm{cl}}}\right\} \delta \phi(x) \\
+ & \left.\int d^{4} x d^{4} y \delta \phi(x) \frac{\delta^{2} \mathcal{A}}{\delta \phi(x) \delta \phi(y)}\right|_{\phi=\phi_{\mathrm{cl}}} \delta \phi(y) \\
& +\mathcal{O}\left((\delta \phi)^{3}\right) .
\end{aligned}
$$

The curly bracket term that is linear in the variation $\delta \phi$ vanishes due to the extremality property of the classical field $\phi_{\mathrm{cl}}$ expressed by the field equation (11). Inserting this expansion into (9), we obtain the approximate expression

$$
\begin{aligned}
& Z[j] \approx \mathcal{N} e^{(i / \hbar)\left\{\mathcal{A}\left[\phi_{\mathrm{cl}}\right]+\int d^{4} x j \phi_{\mathrm{cl}}\right\}} \int \mathcal{D} \delta \phi \\
& \quad \times \exp \left\{\left.\frac{i}{\hbar} \int d^{4} x d^{4} y \delta \phi(x) \frac{\delta^{2} \mathcal{A}}{\delta \phi(x) \delta \phi(y)}\right|_{\phi=\phi_{\mathrm{cl}}} \delta \phi(y)\right\}
\end{aligned}
$$

We now observe that the fluctuations in $\delta \phi$ will be of average size $\sqrt{\hbar}$ due to the $\hbar$-denominator in the Fresnel integrals over $\delta \phi$ in (35). Thus the fluctuations $(\delta \phi)^{n}$ are on the average of relative order $\hbar^{n / 2}$. If we ignore corrections of order $\hbar^{3 / 2}$, the fluctuations remain quadratic in $\delta \phi$ and we may calculate the right-hand side of (35) as

$$
\begin{aligned}
\mathcal{N} e^{(i / \hbar)} & \left\{\mathcal{A}\left[\phi_{\mathrm{cl}}\right]+\int d^{4} x j(x) \phi_{\mathrm{cl}}(x)\right\}\left[\operatorname{det} \frac{\delta^{2} \mathcal{A}}{\delta \phi(x) \delta \phi(y)}\right]_{\phi=\phi_{\mathrm{cl}}} \\
= & \left(\operatorname{det} i G_{0}\right)^{1 / 2} e^{(i / \hbar)\left\{\mathcal{A}\left[\phi_{\mathrm{cl}}\right]+\int d^{4} x j(x) \phi_{\mathrm{cl}}(x)\right\}} \\
& \times e^{(i / \hbar)\left\{i(\hbar / 2) \operatorname{Tr} \log \left[\delta^{2} \mathcal{A} /\left.\delta \phi(x) \delta \phi(y)\right|_{\phi=\phi_{\mathrm{cl}}}\right\}\right.} .
\end{aligned}
$$

Comparing this with the left-hand side of (9), we find that to first order in $\hbar$, the effective action may be recovered by equating

$$
\begin{aligned}
\Gamma[\Phi]+ & \int d^{4} x j(x) \Phi(x)=\mathcal{A}\left[\phi_{\mathrm{cl}}\right]+\int d^{4} x j \phi_{\mathrm{cl}} \\
& +\frac{i \hbar}{2} \operatorname{Tr} \log \frac{\delta^{2} \mathcal{A}}{\delta \phi(x) \delta \phi(y)}\left(\phi_{\mathrm{cl}}\right) .
\end{aligned}
$$


In the limit $\hbar \rightarrow 0$, the trace log term disappears and (37) reduces to the classical action as in (15).

To include the $\hbar$-correction into $\Gamma[\Phi]$, we expand $W[j]$ as

$$
W[j]=W_{0}[j]+\hbar W_{1}[j]+\mathcal{O}\left(\hbar^{2}\right) .
$$

Correspondingly, the field $\Phi$ differs from $\Phi_{\mathrm{cl}}$ by a correction of order $\hbar^{2}$.

$$
\Phi=\phi_{\mathrm{cl}}+\hbar \phi_{1}+\mathcal{O}\left(\hbar^{2}\right)
$$

Inserting this into (37), we find

$$
\begin{aligned}
\Gamma[\Phi]+\int d^{4} x j \Phi & =\mathcal{A}\left[\Phi-\hbar \phi_{1}\right]+\int d^{4} x j \Phi-\hbar \int d^{4} x j \phi_{1} \\
& +\left.\frac{i}{2} \hbar \operatorname{Tr} \log \frac{\delta^{2} \mathcal{A}}{\delta \phi_{a} \delta \phi_{b}}\right|_{\phi=\Phi-\hbar \phi_{1}}+\mathcal{O}\left(\hbar^{2}\right) .
\end{aligned}
$$

Expanding the action up to the same order in $\hbar$ gives

$$
\begin{aligned}
\Gamma[\Phi]= & \mathcal{A}[\Phi]+\hbar\left\{\frac{\delta \mathcal{A}[\Phi]}{\delta \Phi}-j\right\} \phi_{1}+\int d^{4} x j \Phi \\
& +\left.\frac{i}{2} \hbar \operatorname{Tr} \log \frac{\delta^{2} \mathcal{A}}{\delta \phi_{a} \delta \phi_{b}}\right|_{\phi=\Phi}+\mathcal{O}\left(\hbar^{2}\right) .
\end{aligned}
$$

But because of (11), the curly-bracket term is only of order $\mathcal{O}\left(\hbar^{2}\right)$, so that we find the one-loop form of the effective action

$$
\begin{aligned}
\Gamma[\Phi] & =\Gamma_{0}[\Phi]+\Gamma_{1}[\Phi] \\
& =\int d^{4} x\left[\frac{1}{2}\left(\partial \Phi_{a}\right)^{2}-\frac{m^{2}}{2} \Phi_{a}^{2}-\frac{g}{4 !}\left(\Phi_{a}^{2}\right)^{2}\right] \\
& +\frac{i}{2} \hbar \operatorname{Tr} \log \left[-\partial^{2}-m^{2}-\frac{g}{6}\left(\delta_{a b} \Phi_{c}^{2}+2 \Phi_{a} \Phi_{b}\right)\right] .
\end{aligned}
$$

In the special case of a one-component real field, this becomes

$$
\begin{aligned}
\Gamma[\Phi] & =\int d^{4} x\left[\frac{1}{2}(\partial \Phi)^{2}-\frac{m^{2}}{2} \Phi^{2}-\frac{g}{4 !} \Phi^{4}\right] \\
& +\frac{i}{2} \hbar \operatorname{Tr} \log \left[-\partial^{2}-m^{2}-\frac{g}{2} \Phi^{2}\right] .
\end{aligned}
$$

What is the graphical content of the set of all Green functions at this level? For $j=0$, we find that the minimum lies at $\Phi=\Phi^{0} \equiv \Phi_{j}=0$, as in the mean-field approximation. Around this minimum, we may expand the trace $\log$ in powers of $\Phi$, and obtain for $\Gamma^{1}[\Phi]$ the series:

$$
\begin{aligned}
& \frac{i}{2} \hbar \operatorname{Tr} \log \left(-\partial^{2}-m^{2}-\frac{g}{2} \Phi^{2}\right) \\
& \quad=\frac{i}{2} \hbar \operatorname{Tr} \log \left(-\partial^{2}-m^{2}\right)+\frac{i}{2} \hbar \operatorname{Trlog}\left(1+\frac{i}{-\partial^{2}-m^{2}} i g \frac{\Phi^{2}}{2}\right)
\end{aligned}
$$

The second term can be expanded in powers of $\Phi^{2}$ as follows:

$$
-i \frac{\hbar}{2} \sum_{n=1}^{\infty}\left(-i \frac{g}{2}\right)^{n} \frac{1}{n} \operatorname{Tr}\left(\frac{i}{-\partial^{2}-m^{2}} \Phi^{2}\right)^{n} .
$$

If we insert

$$
G_{0}=\frac{i}{-\partial^{2}-m^{2}}
$$

then $\Gamma^{1}[\Phi]$ can be written as

$$
i \frac{\hbar}{2} \operatorname{Tr} \log \left(-\partial^{2}-m^{2}\right)-i \frac{\hbar}{2} \sum_{n=1}^{\infty}\left(-i \frac{g}{2}\right)^{n} \frac{1}{n} \operatorname{Tr}\left(G_{0} \Phi^{2}\right)^{n}
$$

More explicitly, the terms with $n=1$ and $n=2$ read:

$$
\begin{aligned}
& -\frac{\hbar}{2} g \int d^{4} x d^{4} y \delta^{(4)}(x-y) G_{0}(x, y) \Phi^{2}(y) \\
& +i \hbar \frac{g^{2}}{16} \int d^{4} x d^{4} y d^{4} z \delta^{4}(x-z) G_{0}(x, y) \Phi^{2}(y) G_{0}(y, z) \Phi^{2}(z)
\end{aligned}
$$

The expansion terms of (45) for $n \geq 1$ correspond obviously to the Feynman diagrams

Thus the series (45) is a sum of all diagrams formed from one loop and any number of fundamental $\Phi^{4}$-vertices.

This type of loop expansion has been used for many years in the quantum field theory of many-particle systems where it is known as Belyaev expansion [20].

To systematize the entire expansion (45), the trace log term may be pictured by a trivial single-loop diagram without an extra vertex:

$$
i \frac{\hbar}{2} \operatorname{Tr} \log \left(-\partial^{2}-m^{2}\right)=\bigcirc
$$

The first two diagrams in (47) contribute corrections to the vertices $\Gamma^{(2)}$ and $\Gamma^{(4)}$ of (19), (22). The remaining ones produce higher vertex functions and lead to more involved tree diagrams. Note that only the first two corrections are formally divergent, all following loop integrals converge. In momentum space we find from (47)

$$
\begin{aligned}
& \Gamma^{(2)}(q)=q^{2}-m^{2}-\hbar \frac{g}{2} \int \frac{d k^{4}}{(2 \pi)^{4}} \frac{i}{k^{2}-m^{2}+i \eta} \\
& \Gamma^{(4)}\left(q_{i}\right)=g-i \frac{g^{2}}{2} \times \\
& {\left[\int \frac{d^{4} k}{(2 \pi)^{4}} \frac{i}{k^{2}-m^{2}+i \epsilon} \frac{i}{\left(q_{1}+q_{2}-k\right)^{2}-m^{2}+i \eta}+2 \text { perm }\right] .}
\end{aligned}
$$

The convergence of all higher diagrams in the expansion (47) is ensured by the renormalizability of the theory since only up to $n=4$ does one have the possibility to add counter terms of the same form as the original Lagrangian. We may write (49) in euclidean form as

$$
\begin{aligned}
\Gamma^{(2)}(q) & =\left(q^{2}+m^{2}+\hbar \frac{g}{2} D_{1}\right) \\
\Gamma^{(4)}\left(q_{i}\right) & =g-\hbar \frac{g^{2}}{2}\left[I\left(q_{1}+q_{2}\right)+2 \text { perm }\right] .
\end{aligned}
$$


where $D_{1}$ and $I(q)$ are the Feynman integrals

$$
D_{1}=\int d^{4} k_{E} \frac{1}{k_{E}^{2}+m^{2}}
$$

and

$$
I(q)=\int d^{4} k_{E} \frac{1}{\left(k_{E}^{2}+m^{2}\right)} \frac{1}{\left[(k+q)_{E}^{2}+m^{2}\right]} .
$$

The integrals can be calculated in $D$ dimensions by separating them into a directional and a size integral as

$$
\begin{aligned}
\int \vec{d}^{D} k=\int \frac{d^{D} k}{(2 \pi)^{D}} & =\frac{S_{D}}{(2 \pi)^{D}} \int d k k^{D-1} \\
& \equiv \frac{S_{D}}{2} \int d k^{2}\left(k^{2}\right)^{D / 2-1} .
\end{aligned}
$$

We further simplify all calculations by performing a Wick rotation of all energy integrals $\int d k_{0}$ into $i \int_{-\infty}^{\infty} d k_{4}$. Then the integrals $\int d^{4} k$ become what are called euclidean integrals $i \int d^{4} k_{E}$ where $k_{E}^{\mu}=\left(\mathbf{k}, k_{4}\right)$, and $k^{2}=k_{0}^{2}-\mathbf{k}^{2}$ becomes $-k_{E}^{2}=-\left(\mathbf{k}^{2}+k_{4}^{2}\right)$.

By the same token we introduce the euclidean version $\Gamma_{E}[\Phi]=-i \Gamma[\Phi]$ of the effective action (42) whose functional derivatives are vertex functions $\Gamma^{(n)}$ by formulas like (19) and (22).

We further introduce renormalized fields

$$
\phi_{R}(x) \equiv Z_{\phi}^{-1 / 2} \phi(x)
$$

where $Z_{\phi}^{1 / 2}$ is a field renormalization constant. It serves to absorb infinities arising in the momentum integrals. The renormalized vertex functions are obtained by calculating all vertex functions in $D=4-\epsilon$ dimensions and fixing the renormalization constants order by order in perturbation theory. Alternatively, we can add to the bare action suitable counter terms. In either way, we arrive at finite expressions, such as

$$
\begin{aligned}
& \Gamma_{R}^{(2)}(q)=\left\{q^{2}+m^{2}+\frac{\hbar}{2} g \mu^{-\epsilon} S_{D} m^{2}\right. \\
& \left.\times\left[\frac{1}{2} \Gamma(2-\epsilon / 2) \Gamma(-1+\epsilon / 2)\left(\frac{m^{2}}{\mu^{2}}\right)^{-\epsilon / 2}+\frac{1}{\epsilon}\right]\right\}, \\
& \Gamma_{R}^{(4)}\left(q_{i}\right)=g-\hbar \frac{g^{2}}{2}\left[I_{R}\left(q_{1}+q_{2}\right)+2 \text { perm }\right] .
\end{aligned}
$$

where

$$
I_{R}(q)=-\frac{1}{2} S_{D} \mu^{-\epsilon}\left[1+L^{m}(q)+\log \frac{q^{2}}{\mu^{2}}\right]+\mathcal{O}(\epsilon),
$$

with

$$
\begin{aligned}
& L^{m}(q)=\int_{0}^{1} d x \log \left[x(1-x)+\frac{m^{2}}{q^{2}}\right] \\
& =-2+\log \frac{m^{2}}{q^{2}}+\frac{\sqrt{q^{2}+4 m^{2}}}{\sqrt{q^{2}}} \log \frac{\sqrt{q^{2}+4 m^{2}}+\sqrt{q^{2}}}{\sqrt{q^{2}+4 m^{2}}-\sqrt{q^{2}}} .
\end{aligned}
$$

In any regularization scheme, we can also perform subtractions of counter terms which all have the same form as the terms in the original action to remove the divergent parts of the Feynman integrals. In this way we obtain for the the euclidean version $\Gamma_{E}[\Phi]=-i \Gamma[\Phi]$ of the effective action (42) in $D=4-\epsilon$ dimensions the finite subtracted expression

$$
\begin{aligned}
\Gamma_{E}[\Phi] & =\int d^{D} x_{E}\left\{\frac{1}{2}(\partial \Phi)^{2}+\frac{m^{2}}{2} \Phi^{2}+\frac{g}{4 !} \Phi^{4}\right\} \\
& -\frac{\hbar}{2} \operatorname{Tr} \log \left(-\partial^{2}+m^{2}+\frac{g}{2} \Phi^{2}\right) \\
& +\frac{\hbar g}{4} \int \frac{d^{D} q_{E}}{(2 \pi)^{4}} \frac{1}{q_{E}^{2}+m^{2}} \int d^{D} x \Phi^{2}(x) \\
& -\frac{\hbar g^{2}}{16} \int \frac{d^{D} q_{E}}{(2 \pi)^{4}} \frac{1}{\left(q_{E}^{2}+m^{2}\right)^{2}} \int d^{D} x \Phi^{4} .
\end{aligned}
$$

In this formulation, the divergent integrals in the last two terms modify the mass term and the coupling constant. They may be evaluated with any regularization method. A direct evaluation in $4-\epsilon$ dimensions yields

$$
\begin{aligned}
\Gamma_{E}[\Phi] & =\int d^{D} x_{E}\left\{\frac{1}{2}(\partial \Phi)^{2}+\frac{m^{2}}{2} \Phi^{2}+\frac{g}{4 !} \Phi^{4}\right\} \\
& -\frac{\hbar}{2} \operatorname{Tr} \log \left(-\partial^{2}+m^{2}+\frac{g}{2} \Phi^{2}\right) \\
& -{ }^{m} C_{1} \frac{m^{2}}{2} \Phi^{2}-{ }^{g} C_{2} \frac{g^{2}}{4 !} \Phi^{4},
\end{aligned}
$$

where the third line may be written as

$$
-\frac{\hbar g}{4}\left(m^{2}\right)^{1-\epsilon / 2} c_{1} \Phi^{2}+\frac{\hbar g^{2}}{16}\left(m^{2}\right)^{-\epsilon / 2} c_{2} \Phi^{4},
$$

with the constants

$$
\begin{aligned}
& c_{1}=m^{\epsilon-2} \int d^{D} p_{E} \frac{1}{p_{E}^{2}+m^{2}}, \\
& c_{2}=m^{\epsilon} \int \vec{d}^{D} p_{E} \frac{1}{\left(p_{E}^{2}+m^{2}\right)^{2}} .
\end{aligned}
$$

We evaluate these integrals using the formulas 14.

$$
\int d^{D} k_{E} \frac{1}{k_{E}^{2}+m^{2}}=S_{D} \frac{\Gamma(D / 2) \Gamma(1-D / 2)}{2 \Gamma(1)} \frac{1}{\left(m^{2}\right)^{1-D / 2}},
$$

and

$\int d^{D} k_{E} \frac{1}{\left(k_{E}^{2}+m^{2}\right)^{2}}=S_{D} \frac{\Gamma(D / 2) \Gamma(2-D / 2)}{2 \Gamma(1)} \frac{1}{\left(m^{2}\right)^{2-D / 2}} \cdot(67)$

Further, by integrating (66) over $\mathrm{m}^{2}$, we find

$$
\int d^{D} k_{E} \log \left(k_{E}^{2}+m^{2}\right)=S_{D} \frac{\Gamma(D / 2) \Gamma(1-D / 2)}{D \Gamma(1)}\left(m^{2}\right)^{D / 2} \cdot(68
$$

In the so-called minimal subtraction scheme in $4-\epsilon$ dimensions, only the singular $1 / \epsilon$ pole parts of the two 
integrals are selected for the subtraction in (61). In the neighborhood of $\epsilon=0$, (64) and (65) become

$$
\begin{aligned}
& c_{1}=-S_{D} \frac{2}{\epsilon}+\mathcal{O}(\epsilon), \\
& c_{2}=S_{D} \frac{1}{\epsilon}\left(1-\frac{\epsilon}{2}\right)+\mathcal{O}(\epsilon) .
\end{aligned}
$$

Hence we can choose the singular terms in (63)

$$
-\frac{\hbar g \mu^{-\epsilon}}{4} m^{2} S_{D}\left(-\frac{1}{\epsilon}\right) \Phi^{2}, \quad \frac{\hbar g^{2} \mu^{-\epsilon}}{16} S_{D} \frac{1}{\epsilon} \Phi^{4} .
$$

as counter terms. These make the effective action (62) finite for any mass $m$.

Note, however, that an auxiliary mass parameter $\mu$ must be introduced to define these expressions. If the physical mass $m$ is nonzero, $\mu$ can be chosen to be equal to $m$. But for $m=0$, we must use an arbitrary nonzero auxiliary mass $\mu$ as the renormalization scale.

Observe that up to the order $\hbar$, there is no divergence that needs to be absorbed in the gradient term $\int d^{D} x(\partial \Phi)^{2}$ of the effective action (62). These come in as soon as we carry the same analysis to one more loop order.

Let us calculate the effective potential in the critical regime for a constant field $\Phi$ at the one-loop level. It is defined by $v(\Phi)=-\Gamma_{E}[\Phi] / V T$. The argument in the trace log term is now diagonal in momentum space and the calculation reduces to a simple momentum integral. It follows directly from (62) and reads

$$
\begin{aligned}
v(\Phi) & =\frac{m^{2}}{2} \Phi^{2}+\frac{g}{4 !} \Phi^{4}+\frac{\hbar}{2} \int \frac{d^{D} q_{E}}{(2 \pi)^{D}} \log \left(1+\frac{g}{2} \frac{\Phi^{2}}{q_{E}^{2}+m^{2}}\right) \\
& -\frac{\hbar g}{4}\left(m^{2}\right)^{1-\epsilon / 2} c_{1} \Phi^{2}+\frac{\hbar g^{2}}{16}\left(m^{2}\right)^{-\epsilon / 2} c_{2} \Phi^{4}
\end{aligned}
$$

The expansions in powers of $\epsilon$ has an important property which has the direct applications in the description of the strong-coupling limit, i.e., in critical phenomena. For small $\epsilon$, Eq. (57) can be rewritten as

$$
\Gamma_{R}^{(2)}=\left(q_{E}^{2}+m^{2}+\frac{\hbar}{4} \mu^{-\epsilon} g S_{D} \log \frac{m_{E}^{2}}{\mu^{2}}\right)
$$

which is, to the same order in $g$, equal to

$$
\Gamma_{R}^{(2)}(q)=\left[q_{E}^{2}+\left(\frac{m^{2}}{\mu^{2}}\right)^{1+\frac{\hbar}{4} \mu^{-\epsilon} g S_{D}} \mu^{2}\right] .
$$

This means that the vertex function at $q=0$ has a mass term that depends on the mass $m$ of the $\phi$-field via a power law:

$$
\Gamma_{R}^{(2)}(0)=\left(\frac{m^{2}}{\mu^{2}}\right)^{\gamma} \mu^{2}
$$

The power $\gamma$ depends in the coupling strength $g$ like

$$
\gamma=1+\frac{\hbar}{4} \mu^{-\epsilon} g S_{D}
$$

The important point is that this power $\gamma$ is measurable as an experimental quantity called the susceptibility. It is called the critical exponent of the susceptibility.

If the effective action is calculated to order $\hbar^{2}$, then the gradient term in the effective action is modified and becomes

$$
\Gamma[\Phi]=\int d^{d} x \Phi_{R}(x) \Gamma_{R}(\hat{q}) \Phi_{R}(x),
$$

where $\Phi_{R}(x)=Z_{\phi}^{-1 / 2} \Phi(x)$, and $Z_{\phi}$ is the field renormalization constant introduced on (56). It is divergent for $\epsilon \rightarrow 0$ in $D=4-\epsilon$ dimensions. In the critical limit $m^{2} \rightarrow 0$, the renormalization is power-like $\Phi_{R}(x) \rightarrow\left(\mu / \mu_{0}\right)^{-\eta / 2} \Phi(x)$, and Eq. (74) becomes

$$
\Gamma_{R}^{(2)}(q)=-\left[\left(q^{2}\right)^{2-\eta} \mu^{\eta}+\left(\frac{m^{2}}{\mu^{2}}\right)^{\gamma} \mu^{2}\right] .
$$

The power $\eta$ is called the anomalous dimension of the field $\Phi$.

From (78) we extract that the coherence length of the system $\xi$ behaves like

$$
\xi=\mu^{-1}\left(m^{2}\right)^{-\nu}
$$

where

$$
\nu \equiv \gamma /(2-\eta)
$$

is the critical exponent of the coherence length.

Another power behavior is found for $\Gamma_{R}^{(4)}(\mathbf{0})$ :

$$
\begin{aligned}
\Gamma_{R}^{(4)}(\mathbf{0}) \underset{m \rightarrow 0}{\rightarrow} g\left(1+\frac{3}{4} \hbar g \mu^{-\epsilon} S_{D} \log \frac{m^{2}}{\mu^{2}}\right)+\mathcal{O}\left(m^{2}\right) \\
=g\left(\frac{m^{2}}{\mu^{2}}\right)^{\hbar \frac{3}{4} g \mu^{-\epsilon} S_{D}} .
\end{aligned}
$$

Also this power behavior is measurable and defines the critical index $\beta$ via the so-called scaling relation

$$
\gamma-2 \beta \equiv \frac{3}{4} \hbar g \mu^{-\epsilon} S_{D}
$$

so that

$$
\beta=\frac{1}{2}-\frac{1}{4} \hbar g \mu^{-\epsilon} S_{D}
$$

The higher powers of $\Phi$ are accompanied by terms which are more and more singular in the limit $m \rightarrow 0$. We see from the Feynman integrals in (45) that the diagrams in (47) behave like $m^{4-\epsilon-n}$ for $m \rightarrow 0$, and so do the associated effective action terms $\Phi^{n}$.

The coefficients of the dimensionless quantities $\left(\Phi^{2} / \mu^{2-\epsilon}\right) \equiv\left(\tilde{\Phi}^{2}\right)^{n}$ or $\left(g \Phi^{2} / \mu^{2}\right)^{n} \equiv\left(\hat{\Phi}^{2}\right)^{n}$ have the general form $\left(m^{2} / \mu^{2}\right)^{\gamma-2 n \beta}$, so that the effective potential can be written as

$$
v(\Phi)=\mu^{4-\epsilon}\left(\frac{m^{2}}{\mu^{2}}\right)^{\gamma} \frac{\Phi^{2}}{\mu^{2-\epsilon}} f(x)
$$


with

$$
x \equiv\left(\frac{m^{2}}{\mu^{2}}\right)^{-2 \beta} \frac{\Phi^{2}}{\mu^{2-\epsilon}} \equiv t^{-2 \beta} \frac{\Phi^{2}}{\mu^{2-\epsilon}}=t^{-2 \beta} \tilde{\Phi}^{2},
$$

where we have abbreviated

$$
t \equiv \frac{m^{2}}{\mu^{2}}
$$

For small $\Phi$, the function $f(x)$ has a Taylor expansion in even powers of $\Phi$, corresponding to the diagrams in Eq. (47):

$$
f(x)=1+\sum_{n=1}^{\infty} f_{n} x^{n}=1+\sum_{n=1}^{\infty} f_{n}\left(t^{-2 n \beta} \tilde{\Phi}^{2}\right)^{n} .
$$

If we start the sum at $n=-1$, we also get the general form of the vacuum energy

$$
v(0)=\mu^{4-\epsilon}\left(\frac{m^{2}}{\mu^{2}}\right)^{\gamma+2 \beta}
$$

The exponent $\gamma+2 \beta$ is equal to $D \nu$, where $\nu$ is the exponent defined in (80).

By differentiating this energy twice with respect to $\mathrm{m}^{2}$, we obtain the temperature behavior of the specific heat

$$
C \propto\left(\frac{m^{2}}{\mu^{2}}\right)^{D \nu-2}=\left(\frac{m^{2}}{\mu^{2}}\right)^{-\alpha}
$$

which yields the important critical exponent $\alpha=2-$ $D \nu$ that governs the singularity of the famous $\lambda$-peak in superfluid helium at $T_{c} \approx 2.7$ Kelvin, the most accurately determined critical exponent in many-body systems by a measurement in a satellite [19].

We can also rewrite (84) in the form

$$
v(\Phi)=\mu^{4-\epsilon} \frac{S_{D}}{\lambda}\left(\frac{m^{2}}{\mu^{2}}\right)^{\gamma} \frac{g \Phi^{2}}{\mu^{2}} \bar{f}(y),
$$

where

$$
\bar{f}(y)=1+\sum_{n=1}^{\infty} \bar{f}_{n} y^{n}=1+\sum_{n=1}^{\infty} \bar{f}_{n}\left(t^{-2 \beta}\left(\frac{g \Phi^{2}}{\mu^{2}}\right)\right)^{n} .
$$

and

$$
y \equiv \frac{\lambda}{S_{D}} x=g \mu^{-\epsilon} x=\left(\frac{m^{2}}{\mu^{2}}\right)^{-2 \beta}\left(\frac{g \Phi^{2}}{\mu^{2}}\right)=t^{-2 \beta} \hat{\Phi}^{2}
$$

In the limit $m^{2} \rightarrow 0$, the expansion (87) and (91) are divergent since the the coefficients grow like $n$ !. A sum can nevertheless be calculated with the technique of Variational Perturbation Theory (VPT) developed in the textbook 14].

\section{MASSLESS THEORY AND WIDOM SCALING}

Let us evaluate the zero-mass limit of $v(\Phi)$. Since $m^{2}$ always accompanies the coupling strength in the denominator, the limit $m^{2} \rightarrow 0$ is equivalent to the limit $g \rightarrow \infty$, i.e., the strong-coupling limit.

The strong-coupling limit deserves special attention. The theory in this limit is referred to the critical theory. This name reflects the relevance of this limit for the behavior of physical systems at a critical temperature where fluctuations are of infinite range.

We shall see immediately that for large $y, f(y)$ behaves like a pure power of $y: f(y) \rightarrow y^{(\delta-1) / 2}$, so that

$$
v(\Phi) \rightarrow \mu^{4-\epsilon} \frac{1}{4 !} \frac{S_{D}}{\lambda}\left(\frac{g \Phi^{2}}{\mu^{2}}\right)^{(\delta+1) / 2} .
$$

Since without fluctuation corrections, $\delta=3$, this reduces properly to the mean-field potential $g \Phi^{4} / 4$ !.

With this leading large $\Phi$-behavior, we can rewrite the general form (90) of the potential also as a socalled Widom scaling expression depending on $y^{-1 / 2 \beta} \propto$ $m^{2} / \Phi^{1 / \beta}[11]$ :

$$
v(\Phi) \propto \Phi^{\delta+1} w\left(m^{2} / \Phi^{1 / \beta}\right) .
$$

From this effective potential we may derive the general Widom form of the equation of state. After adding a source term $H \Phi$ and going to the extremum, we obtain $H(\Phi)=\partial v(\Phi) / \partial \Phi$ with the general behavior

$$
H(\Phi) \propto \Phi^{\delta} h\left(m^{2} / \Phi^{1 / \beta}\right) .
$$

Recalling (94), we expect the general form of the potential (84) to be

$$
v(\Phi) \rightarrow \mu^{4-\epsilon} \frac{1}{4 !} \frac{S_{D}}{\lambda}\left(\frac{g \Phi^{2}}{\mu^{2}}\right)^{(\delta+1) / 2} \hat{w}(\tau),
$$

where $\tau \equiv\left(m^{2} / \mu^{2}\right) /\left(\Phi / \mu^{1-\epsilon / 2}\right)^{1 / \beta} \equiv t /\left(\tilde{\Phi}^{2}\right)^{1 / \beta}$.

For small $m^{2}, \hat{w}(\tau)$ has a series expansion in powers of $\tau^{\omega \nu}$ :

$$
\left.\hat{w}(\tau)=1+c_{1} \tau^{\omega \nu}+c_{2} \tau^{2 \omega \nu}+\ldots\right),
$$

or since $t=\mu \xi^{-1 / \nu}$ :

$$
\left.\hat{w}(\tau)=1+\bar{c}_{1} \xi^{-\omega} \Phi^{-\omega \nu / \beta}+\bar{c}_{2} \xi^{-2 \omega} \Phi^{-2 \omega \nu / \beta}+\ldots\right) .
$$

Here $\omega$ is the Wegner exponent [17] that governs the approach to scaling. Its numerical value is close to 0.8 [22].

Differentiating (96) with respect to $\Phi$ yields the following leading contribution to $H$ :

$$
\begin{aligned}
H & =\partial_{\Phi} v(\Phi)=\frac{\delta+1}{4 !} \mu^{2}\left(\frac{g \Phi^{2}}{\mu^{2}}\right)^{(\delta-1) / 2} \\
& =\frac{\delta+1}{4 !} \mu^{2}\left(\frac{\lambda \tilde{\Phi}^{2}}{S_{D}}\right)^{(\delta-1) / 2},
\end{aligned}
$$


where $\delta-1=\gamma / \beta$.

Note that the effective potential remains finite for $m=$ 0 . Then, $v(\Phi)$ becomes

$v(\Phi)=\frac{g}{4 !} \Phi^{4}+\frac{\hbar}{2} \int \frac{d^{D} q}{(2 \pi)^{D}} \log \left(1+\frac{g}{2} \frac{\Phi^{2}}{q^{2}}\right)+\frac{\hbar g^{2} \mu^{-\epsilon}}{16} \frac{S_{D}}{\epsilon} \Phi^{4} \cdot(100)$

It displays an important feature: When expanding the logarithm in powers of $\Phi$, the expansion terms correspond to increasingly divergent Feynman integrals

$$
\int \frac{d^{D} q}{(2 \pi)^{D}} \frac{1}{\left(q^{2}\right)^{n}}
$$

Contrary to the previously regularized divergencies coming from the large- $q^{2}$ regime, these divergencies are due to the $q=0$-singularity of the massless propagators $G_{0}(q)=i / q^{2}$. This means that they are IR-singularities. Let us verify that the effective potential remains indeed finite for $m=0$. Performing the momentum integral in (100) the potential becomes

$$
v(\Phi)=\frac{g}{4 !} \Phi^{4}+\frac{\hbar}{4} S_{D}\left(-\frac{1}{\epsilon}\right)\left(\frac{g}{2} \Phi^{2}\right)^{2-\frac{\epsilon}{2}}+\frac{\hbar g^{2}}{16} S_{D} \mu^{-\epsilon} \frac{1}{\epsilon} \Phi^{4} .
$$

With the goal of expanding this for small $\epsilon$-expansion, we divide the coupling constant and field by a scale parameter involving $\mu$. Then $g \mu^{-\epsilon}$ and $\Phi / \mu^{1-\epsilon / 2}$ are dimensionless quantities, in terms of which

$$
\begin{aligned}
v(\Phi) & =\mu^{4-\epsilon}\left[\frac{g \mu^{-\epsilon}}{4 !}\left(\frac{\Phi}{\mu^{1-\epsilon / 2}}\right)^{4}+\frac{\hbar}{4} S_{D}\left(-\frac{1}{\epsilon}\right)\right. \\
& \left.\times\left(\frac{g \mu^{-\epsilon}}{2} \frac{\Phi^{2}}{\mu^{2-\epsilon}}\right)^{2-\frac{\epsilon}{2}}\right]+\frac{\hbar g^{2}}{16} \frac{\mu^{-2 \epsilon}}{\epsilon} S_{D}\left(\frac{\Phi}{\mu^{1-\epsilon / 2}}\right)^{4} .
\end{aligned}
$$

If we use the dimensionless coupling constant

$$
\lambda \equiv S_{D} \hbar g \mu^{-\epsilon}
$$

and the reduced field $\tilde{\Phi} \equiv \Phi / \mu^{1-\epsilon / 2}$ as new variables, then

$$
\begin{aligned}
v(\Phi)=\frac{\mu^{4-\epsilon}}{\hbar S_{D}} & {\left[\frac{\lambda}{4 !} \tilde{\Phi}^{4}+\frac{1}{4}\left(-\frac{1}{\epsilon}\right)\left(\frac{\lambda \tilde{\Phi}^{2}}{2}\right)^{2}\right.} \\
\times & \left.\left(1-\frac{\epsilon}{2} \log \frac{\lambda \tilde{\Phi}^{2}}{2}\right)+\frac{\lambda^{2}}{16 \epsilon} \tilde{\Phi}^{4}\right\} .
\end{aligned}
$$

To zeroth order in $\epsilon$, the prefactor is equal to $\mu^{4} 8 \pi^{2}$. Thus the massless limit of the effective potential is well defined in $D=4$ dimensions. There is, however, a special feature: The finiteness is achieved at the expense of introducing the extra parameter $\mu$.

The most important property of the critical potential is that it cannot be expanded in integer powers of $\Phi^{2}$.
Instead, the expression (104) can be rewritten, correctly up to order $\lambda^{2}$, as

$$
\begin{aligned}
v(\Phi) & =\frac{\mu^{4-\epsilon}}{S_{D}} \frac{1}{6}\left\{\left(\frac{\lambda}{2} \tilde{\Phi}^{2}\right)^{2}+\frac{3}{8}\left(\frac{\lambda}{2} \tilde{\Phi}^{2}\right)^{2} \log \frac{\lambda}{2} \tilde{\Phi}^{2}\right\} \\
& =\frac{\mu^{4-\epsilon}}{S_{D}} \frac{1}{6 \lambda}\left(\frac{\lambda}{2} \tilde{\Phi}^{2}\right)^{2+\frac{3}{4} \lambda}+\mathcal{O}\left(\lambda^{3}\right) .
\end{aligned}
$$

This is the typical power behavior of the critical interaction. The power of $\tilde{\Phi}$ defines the critical exponent of the interaction $1+\delta$, so that up to the first order in the coupling strength:

$$
\delta=3+\frac{3}{2} \lambda .
$$

At the mean-field level, $\delta$ is equal to 3

\section{CRITICAL COUPLING STRENGTH}

What is the coupling strength $\lambda$ in the critical regime? The counter term proportional to $\Phi^{4}$ in Eq. (100) implies that the use of the renormalized coupling constant in the subsequent subtracted expressions. This corresponds to the use of a bare coupling constant $g_{B}$ instead of $g$ in the original action (17). The relation between the two to this one-loop order is

$$
g_{B}=g+\frac{3}{2} \hbar g^{2} \mu-\epsilon \frac{S_{D}}{\epsilon}+\ldots .
$$

For a given bare interaction strength $g_{B}$, the renormalized coupling depends on the parameter $\mu$ chosen for the renormalization procedure. Equivalently we may imagine having defined the field theory on a fine spatial lattice with a specific small lattice spacing $a \propto 1 / \mu$, and the renormalized coupling constant will depend on the choice of $a$.

If we sum an infinite chain of such corrections, we obtain a geometric sum that is an expansion of the equation

$$
g_{B}=\frac{g}{1-\frac{3}{2} \hbar g \mu^{-\epsilon} \frac{S_{D}}{\epsilon}},
$$

which has (107) as its first expansion term. Equivalently we may write

$$
\frac{1}{\hbar g_{B} \mu^{-\epsilon}}=\frac{1}{\hbar g \mu^{-\epsilon}}-\frac{3}{2} \frac{S_{D}}{\epsilon}+\ldots .
$$

In this equation, we can go to the strong-coupling limit $g_{B} \rightarrow \infty$ by taking $\mu$ to the critical limit $\mu \rightarrow 0$, where we find that the renormalized coupling has a finite value

$$
\frac{1}{\hbar g \mu^{-\epsilon}}=\frac{3}{2} \frac{S_{D}}{\epsilon}+\ldots
$$


For the dimensionless coupling constant (103), this amounts to the strong-coupling limit

$$
\lambda \equiv \hbar g \mu^{-\epsilon} S_{D} \rightarrow \frac{2}{3} \epsilon+\ldots,
$$

with the omitted terms being of higher order in $\epsilon$. The approach to this limit starting from small coupling is obtained from (108) to be

$$
\hbar g \mu^{-\epsilon}=\frac{1}{\frac{1}{\hbar g_{B} \mu^{-\epsilon}}+\frac{3}{2} \frac{S_{D}}{\epsilon}} .
$$

At small bare coupling constant, this starts out with the renormalized expression that is determined by the $s$ wave scattering length $\hbar g \mu^{-\epsilon}=6 \times 4 \pi \hbar^{2} a_{s} / M$. In the strong-coupling limit of $g_{B}$ or the critical limit $\mu \rightarrow 0$, the value (110) is reached.

For the critical exponents $\gamma, \beta, \delta$ in (76), (83), (106), the strong-coupling limits are

$$
\gamma=1+\frac{1}{6} \epsilon, \quad \beta=\frac{1}{2}-\frac{1}{6} \epsilon, \quad \delta=3+\epsilon .
$$

They are approached for finite $g_{B}$ like

$$
\gamma=1+\frac{1}{4} \hbar g \mu^{-\epsilon}, \quad \beta=\frac{1}{2}-\frac{1}{4} \hbar g \mu^{-\epsilon},
$$

with the just discussed $g_{B}$-behavior of $g$.

If the omitted terms in (111) are calculated for all $N$ and to higher loop orders one finds $\lambda=2 g^{*}$, where $g^{*}$ is the strong-coupling limit $g_{B} \rightarrow \infty$ of the series in Eq. (15.18) in the textbook [14]]. The other critical exponents may be obtained from the $\bar{g}_{B} \rightarrow \infty$-limit of similar expansions for $\nu, \eta$ into $\alpha=2-\nu D, \beta=\nu(D-2+\eta)$, $\gamma=\nu(2-\eta), \delta=(D+2-\eta) /(D-2+\eta)$. These can be extracted from Chapter 15 in Ref. [14].

All these series are divergent, but can be resummed for $\epsilon=1$ and $\bar{g}_{B} \rightarrow \infty$. The series for $\bar{g}$ yields for $N=2$ the value $\bar{g} \rightarrow g^{*} \approx 0.503$ (see Fig. 17.1 in [14]). The typical dependence of $\bar{g}$ on $\bar{g}_{B}$ is plotted in Fig. 20.8 of Ref. [14]. For $\nu$ and $\eta$ the plots are shown in Figs. 20.9 and 20.10 , and we leave it to the reader to compose from these the dependence of $\alpha, \beta, \delta$ as functions of $\bar{g}_{B}$.

It should be pointed out that the potential in the first line of (105) has another minimum away from the origin at

$$
\frac{\lambda}{3 !} \tilde{\Phi}^{3}+\frac{\lambda^{2}}{8} \tilde{\Phi}^{3}\left(\log \frac{\lambda}{2} \tilde{\Phi}^{2}-\frac{1}{2}\right)+\frac{\lambda^{2}}{16} \tilde{\Phi}^{3}=0 .
$$

This is solved by

$$
\lambda \log \frac{\lambda}{2} \tilde{\Phi}^{2}=-\frac{4}{3}
$$

or

$$
\tilde{\Phi}^{2}=\frac{2}{\lambda} e^{-4} 3 \lambda .
$$

However such a solution found for small $\lambda$ cannot be trusted. The higher loops to be discussed later and neglected up to this point will produce more powers of $\lambda \log \left(\lambda \tilde{\Phi}^{2} / 2\right)$, and the series cannot be expected to converge at such a large $\lambda$. As a matter of fact, the approximate exponentiation performed in (105) does not show this minimum and will be seen, via the methods to be described later, to be the correct analytic form of the potential to all orders in $\lambda$ for small enough $\epsilon$ and $\lambda$.

If we want to apply the formalism to a Bose-Einsteincondensate we must discuss the case of a general $\mathrm{O}(N)$ symmetric version of the effective potential based on the action (41), and insert into it the number $N=2$. The equation for the bare coupling constant is then

$$
g_{B}=g+\frac{N+8}{6} \hbar g^{2} \mu^{-\epsilon} \frac{S_{D}}{\epsilon}+\ldots,
$$

rather than (107), so that the strong-coupling limit (111) becomes

$$
\lambda \equiv S_{D} \hbar g \mu^{-\epsilon} \rightarrow \frac{6}{N+8} \epsilon+\ldots,
$$

with the other critical exponents (113):

$\gamma=1+\frac{N+2}{2(N+8)} \epsilon, \beta=\frac{1}{2}-\frac{3}{2(N+8)} \epsilon, \delta=3+\frac{9}{N+8} \epsilon$.

For the coupling constant $g_{S}$ defined in (25) the strongcoupling limit (119) reads

$$
g_{S} \rightarrow 2 \frac{1}{4 !} \frac{\lambda}{S_{D}} \mu^{\epsilon}=\frac{(4 \pi)^{2}}{4 !} \lambda \mu^{\epsilon}=\frac{(4 \pi)^{2}}{12} g^{*} \mu^{\epsilon},
$$

with $g^{*} \approx 0.503$ for $N=2$.

If we carry the loop expansion to higher order in $\hbar$, we find for the renormalized $\bar{g}=\hbar g_{B} \mu^{-\epsilon} / 2 S_{D}$ as function of the bare coupling $\bar{g}_{B}=\hbar g_{B} \mu^{-\epsilon} / 2 S_{D}$ the perturbation expansion [see Eq. (15.18) in Ref. 14]. See there also the higher expansion terms]

$$
\begin{aligned}
\frac{\bar{g}}{\bar{g}_{B}}=1- & \bar{g}_{B} \frac{8+N}{3} \epsilon^{-1}+\bar{g}_{B}^{2}\left\{\frac{(8+N)^{2}}{9} \frac{1}{\epsilon^{2}}+\frac{14+3 N}{6} \frac{1}{\epsilon}\right\} \\
+ & \bar{g}_{B}^{3}\left\{-\frac{(8+N)^{3}}{27} \frac{1}{\epsilon^{3}}-4 \frac{(8+N)(14+3 N)}{27} \frac{1}{\epsilon^{2}}\right. \\
& \left.-\frac{\left[2960+922 N+33 N^{2}+(2112+480 N) \zeta(3)\right]}{648} \frac{1}{\epsilon}\right\}+\ldots
\end{aligned}
$$

Given this dependence of $\bar{g}$ on the bare coupling constant $\bar{g}_{B}$, we find for $\gamma$ the expansion

$$
\begin{aligned}
\gamma\left(\bar{g}_{B}\right) & =\frac{\bar{g}}{6}(N+2)-5 \frac{\bar{g}^{2}}{36}(N+2)+\frac{\bar{g}^{3}}{72}(N+2)(5 N+37) \\
& -\frac{\bar{g}^{4}}{15552}(N+2)\left[-N^{2}+7578 N+31060\right. \\
& \left.+48 \zeta(3)\left(3 N^{2}+10 N+68\right)+288 \zeta(4)(5 N+22)\right]+\ldots,
\end{aligned}
$$

where $\bar{g}$ is replaced by (122).

The expression $\left(\mathrm{m}^{2} / \mu^{2}\right)^{\gamma}$ in the two-point function can be replaced by an expansion of $\mathrm{m}^{2} / \mathrm{m}_{B}^{2}$ in powers of $\bar{g}_{B}$ 
that can be taken from Eq. (15.15) in [14], again with $\bar{g}$ replaced by (122). This expansion can be resummed by Variational Perturbation Theory to obtain a curve of the type in Fig. 20.8-20.10. This permits us to relate $t=m^{2} / \mu^{2}$ directly to $\mu^{-\epsilon} g_{B}$.

\section{RESUMMING THE EFFECTIVE POTENTIAL}

According to Eq. (90), the effective action below in the condensed phase with negative $m^{2}$ has the general form

$$
\frac{v(\Phi)}{\mu^{4-\epsilon}}=t^{\gamma} \frac{\Phi^{2}}{\mu^{2-\epsilon}}\left(\bar{f}_{0}+\bar{f}_{1} y+\ldots\right),
$$

where

$$
y=t^{-2 \beta} g \Phi^{2} / \mu^{2}=t^{-2 \beta} \hat{\Phi}^{2}
$$

From Eq. 91 we determine $\bar{f}_{0}=\bar{f}_{1}=1$. For small $t, y$ becomes large, and we must convert the small-y expansion into a large- $y$ expansion.

Near the strong-coupling limit, the Widom function (94) has an expansion in powers of $\left(m^{2}\right)^{\omega / \nu} \propto \xi^{-\omega}$ which contains the Wegner critical exponent $\omega \approx 0.8$ governing the approach to scaling [22]. The general expansion for strong couplings is

$\frac{v(\Phi)}{\mu^{4-\epsilon}}=t^{\gamma} \frac{\Phi^{2}}{\mu^{2-\epsilon}}\left(t^{-2 \beta} \hat{\Phi}^{2}\right)^{(\delta-1) / 2}\left(b_{0}+\sum_{m=1}^{\infty} \frac{b_{m}}{\left(t^{-2 \beta} \hat{\Phi}^{2}\right)^{m \omega \nu / 2 \beta}}\right)$,

We may derive this from the rules of VPT in [14]. First we rewrite a variational ansatz for the right-hand side of Eq. (124) with the help of a dummy parameter $\kappa=1$ as

$$
w_{N}=\mu^{2} t^{\gamma} \Phi^{2} \kappa^{p}\left(\bar{f}_{0}+\bar{f}_{1} \frac{y}{\kappa^{q}}+\ldots\right) .
$$

Next we exchange $\kappa^{p}$ by the identical expression ${\sqrt{K^{2}+g r}}^{p}$, where $r \equiv\left(k^{2}-K^{2}\right) / K^{2}$. After this we form $w_{1}$ by expanding $w_{N}$ up to order $g$, and setting $\kappa=1$. This leads to

$$
\begin{aligned}
w_{1} & =\mu^{2} t^{\gamma} \Phi^{2} K^{p}\left[\bar{f}_{0}\left(1-\frac{p}{2}+\frac{p}{2} \frac{1}{K^{2}}\right)+\bar{f}_{1} \frac{y}{K^{q}}\right] \\
& =\mu^{2} t^{\gamma} \Phi^{2} W_{1}(y) .
\end{aligned}
$$

The last term in the first line shows that for large $y, K$ has to grow like

$$
K \propto y^{1 / q}
$$

We now extremize $w_{1}$ with respect to $K$ and find that the derivative $d w_{1} / d K$ has to vanish, i.e.,

$$
t^{\gamma} \Phi^{2} K^{p-1} \frac{p(2-p)}{2}\left[\bar{f}_{0}\left(1-\frac{1}{K^{2}}\right)-\bar{f}_{1} c \frac{y}{K^{q}}\right]=0,
$$

where

$$
c \equiv \frac{2(p-q)}{p(p-2)} \approx 0.32 .
$$

In the free-particle limit $y \rightarrow 0$, the solution is $K(0)=1$, and

$$
w_{1}=\mu^{2} t^{\gamma} \Phi^{2} \bar{f}_{0} .
$$

The last term in (130) shows once more that for large $y, K$ will be proportional to $y^{1 / q}$. Moreover, it allows to sharpen relation (129) to the large- $y$ behavior

$$
K \rightarrow K_{\mathrm{as}}(y)=(c y)^{1 / q} \approx 0.648 y^{0.381} .
$$

Then the leading large- $y$ behavior of (108) is $\Phi^{2}(c y)^{p / q} \propto$ $\left(\Phi^{2}\right)^{p+1}$.

The first correction to the large- $y$ behavior comes from the second term in the brackets of (130), which by (125) should behave like

$$
K^{2} \rightarrow\left(c t^{-2 \beta} g \Phi^{2} / \mu^{2}\right)^{\omega \nu / 2 \beta} \approx\left(c t^{-2 \beta} g \Phi^{2} / \mu^{2}\right)^{0.76} .(13
$$

Comparing this with (110) and (129), we find $p=2(2-$ $\eta) / \omega$ and $q=4 \beta / \omega \nu$. For the $N=2$ universality class these have the numerical values $p \approx 4.92$ and $q \approx 4 \times$ $0.32 /(0.8 \times 0.66) \approx 2.63$. Solving (130), we see that for small $y, K(y)$ has the diverging expansion

$$
K(y)=1+0.32 y-0.165967 y^{2}+0.155806 y^{3}+\ldots,
$$

so that $W_{1}$ has the diverging expansion

$$
W_{1}=1+y+0.154436 y^{3}+\ldots .
$$

From Eq. (93), we know that the power $p+1$ must be equal to $(\delta+1) / 2$, so that

$$
y^{p / q}=y^{(\delta-1) / 2}=y^{(2-\eta) \nu / 2 \beta} \approx y^{1.87} .
$$

If we insert (129) into (108), the extremal variational energy is

$$
w_{1}(y)=\mu^{2} t^{\gamma} \Phi^{2} K^{p}\left(1-\frac{p}{2}+\frac{y}{K^{q}}\right) .
$$

where $K=K(y)$ is a function $y$ which is plotted in Fig. 1.

For large $y$, where $K(y)$ has the limiting behavior (133), $W_{1}$ becomes

$$
\begin{aligned}
W_{1}(y) & \rightarrow W_{\text {as }}(y)=K^{p}\left(1-\frac{p}{2}+\frac{1}{c}\right) \\
& \approx 0.197 y^{1.87} .
\end{aligned}
$$

Near the limit, the corrections to (133) are

$$
\begin{aligned}
K(y) & =K_{\mathrm{as}}(y)\left(1+\sum_{m=1}^{\infty} \frac{h_{m}}{y^{m \omega \nu / 2 \beta}}\right) \\
& \approx 0.648 y^{0.381}\left(1+\sum_{m=1}^{\infty} \frac{h_{m}}{y^{0.761 m}}\right) .
\end{aligned}
$$



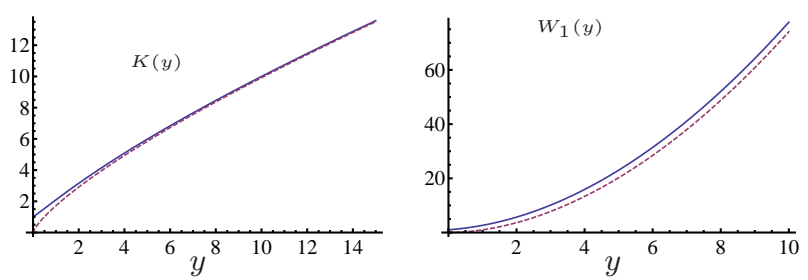

Figure 1: Solution of the variational equation (130) for $\bar{f}_{1}=1$. The dotted curves show the pure large- $y$ behavior.

with $h_{1} \approx 0.909, h_{2} \approx-0.155, \ldots$. Inserting this into (128), we find

$$
\begin{aligned}
W_{1}(y) & =W_{\mathrm{as}}(y)\left(1+\sum_{m=1}^{\infty} \frac{b_{m}}{y^{m \omega \nu / 2 \beta}}\right) \\
& \approx 0.197 y^{1.87}\left(1+\sum_{m=1}^{\infty} \frac{b_{m}}{y^{0.761 m}}\right) .
\end{aligned}
$$

with $b_{1} \approx 3.510, b_{2} \approx 4.65248, \ldots$.

\section{FRACTIONAL GROSS-PITAEVSKII EQUATION}

We now extremize the effective action (62) with the two-loop corrected quadratic term (74). We consider the case of $N=2$ where $\Phi^{2}=\Psi^{*} \Psi$. Then we take the effective potential (138) with the extremal $K=K(y)$ as a function $y$ plotted in Fig. 1. From this we form the derivative $\partial w_{1}(y) / \partial \Psi^{*}$ and obtain the time-independent fractional Gross-Pitaevskii equation:

$$
\left(\hat{\mathbf{p}}^{2}\right)^{1-\eta / 2} \Psi+\frac{\partial w_{1}(y)}{\partial \Psi^{*}}=0 .
$$

If we use the weak-coupling limit of $w_{1}(y)$ and the gradient term, this reduces to the ordinary time-independent Gross-Pitaevskii equation

$$
\left[-\frac{\hbar^{2}}{2 M} \nabla^{2}-\mu+g_{S} \Psi^{\dagger} \Psi\right] \Psi(x)=0 .
$$

In a harmonic trap $\mu$ is replaced by $\mu+M \omega^{2} \mathbf{x}^{2} / 2$. Recalling the relation $m^{2}=-2 M \mu$ one has $m^{2}=$ $-2 M M \omega^{2} R_{c}^{2}\left(1-R^{2} / R_{c}^{2}\right)$. The oscillator energy $M \omega^{2} R_{c}^{2}$ corresponds to a length scale $\ell_{\mathcal{O}}$ by the relation $M \omega^{2} R_{c}^{2}=\hbar^{2} / M \ell_{\mathcal{O}}^{2}$, so that $m^{2}$ may be written as $m^{2}=\mu^{2}\left(R^{2} / R_{c}^{2}-1\right)$ with $\mu=1 / \ell_{\mathcal{O}}$.

In the strong-coupling limit, however, we arrive at the time-independent fractional Gross-Pitaevskii equation:

$$
\left[\left(\hat{\mathbf{p}}^{2}\right)^{1-\eta / 2}+\frac{\delta+1}{4 \mu^{\eta}} g_{c}|\Psi(\mathbf{x})|^{\delta-1}\right] \Psi(\mathbf{x})=0 .
$$

By using the full effective action for all coupling strengths and masses $m^{2}$ we can calculate the properties of the condensate at an coupling strength. Before reaching the strong-coupling limit, we may use Eq. (142) to calculate the field strength as a function of $g_{S}$. Then the critical exponents $\eta, \alpha, \beta, \delta$ have not yet reached their strong-coupling values by must be replaced by the $\bar{g}_{B^{-}}$ dependent precritical values calculated from (122) and the corresponding equations for $\eta, \alpha, \beta, \delta$.

In a trap, the mass term becomes weakly spacedependent. If the trap is rotationally symmetric, then $m^{2}$ will depend on $R=|\mathbf{x}|$ and the time-independent Gross-Pitaevskii equation has to be solved with $m^{2}(R) \propto$ $1-R^{2} / R_{c}^{2}$. More specifically, the bare coupling constant on the right-hand side has to be determined in such a way that $m^{2} / m_{B}^{2}$ has the experimental size. If the experiments are performed in an external magnetic field $B$ the $s$-wave scattering length $a_{s}$ has an enhancement factor $\left(B / B_{c}-1\right)^{-1}$ and Eq. (122) can again be used. We can then calculate the density profile quite easily in the Thomas-Fermi approximation as done in Ref. [23].

In a rotating $\mathrm{BEC}$ we can calculate the different forms of the density profiles of vortices for various coupling strengths which can be varied from weak to strong by subjecting the BEC to different magnetic fields, raising it from zero up to the Feshbach resonance. The profiles are shown in Fig. 22).

In addition, the central region is depleted (see Fig. 2).
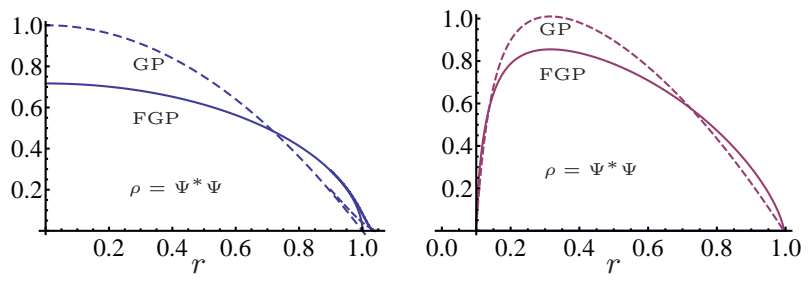

Figure 2: Condensate density from Gross-Pitaevskii equation (24) (GP,dashed) and its fractional version (144 (FGP), both in Thomas-Fermi approximation where the gradients are ignored. The FGP-curve shows a marked depletion of the condensate. On the right hand, a vortex is included. The zeros at $r \approx 1$ will be smoothened by the gradient terms in 24) and (144), as shown on the left-hand plots without a vortex. The curves can be compared with those in Ref. 24 28].

\section{SUMMARY}

We have shown that the expansion of the effective action of a $\phi^{4}$-theory in even powers of the field strength $\Phi=\langle\phi\rangle$ can be be resummed to obtain an expression that is valid for any field strength, even in the the strongcoupling limit. It has the phenomenological scaling form once proposed by Widom, and can be used to calculate the shape of a BEC up to the Feshbach resonance, with and without rotation. 
* Electronic address: h.k@fu-berlin.de

[1] S.N. Bose, Z. Phys. 26, 178 (1924). J.Phys. USSR, 2, 23 (1947).

[2] A. Einstein, Sitzber. Kgl. Preuss. Akad. Wiss. (1924), p.261; (1926), p.3. See the story in A. Pais, Subtle is the Lord. The Life of Albert Einstein, Clarendon Press, Oxford, 1982.

[3] N.N. Bogoliubov, Z. Phys. 26, 178 (1924).

[4] There are many excellent textbooks on this subject, for example

A. Griffin, D.W. Snoke, and S. Stringari, Bose-Einstein Condensation, Cambridge University Press, Cambridge, 1996; S. Martelucci, A.N. Chester, A. Aspect, and M. Inguscio, Bose-Einstein Condensation and Atom Lasers, Kluwer, N.Y., 2002;

C.J. Pethick, H. Smith, Bose-Einstein Condensation in Dilute Atomic Gases, Cambridge University Press, Cambridge, 2008; Bose-Einstein Condensation, Clarendon Press, Oxford,2003.

[5] L. Pitaevskii and S. Stringari, Bose-Einstein Condensation, Clarendon Press, Oxford,2003.

V.I. Yukalov, Laser Phys. 19, 1 (2009) (arXiv:0901.0636). See also Chapter 7 in [6] (http://klnrt.de/b5).

[6] A first-quantized treatment of the many-boson condensation problem can be found in Chapter 7 of the textbook

H. Kleinert, Path Integrals in Quantum Mechanics, Statistics, Polymer Physics, and Financial Markets, World Scientific, Singapore, 2006. (http://klnrt.de/b5).

[7] For a good review see, for example, the 2006 MIT thesis of M. Zwierlein, (http://cua.mit.edu).

[8] J. Zinn-Justin, Quantum field theory and critical phenomena, Oxford, Clarendon Press (2002).

[9] E.C.G. Stueckelberg and A. Petermann, Helv. Phys. Acta 26, 499 (1953).

[10] M. Gell-Mann and F.E Low, Physical Review 95, 1300 (1954).

[11] B. Widom, J. Chem. Phys. 43, 3892, 3898 (1965).

[12] H.E.Stanley, Introduction to Phase Transitions and Critical Phenomena, Clarendon Press, Oxford,1071.

[13] Leo Kadanoff, Statistical Physis. Statics, Dynamics and Renormalization, World Scientific, Singapore, 2000.

[14] H. Kleinert and V. Schulte-Frohlinde, Critical Properties of $\phi^{4}$-Theories, World Scientific, Singapore 2001 (http://klnrt.de/b8).

[15] Such methods go back to
R.P. Feynman, Statistical Mechanics, (Benjamin, Reading, MA, 1972, R.P. Feynman and H. Kleinert, Phys. Rev. A B 34, 5080 (1986). This first-order method was extended to arbitrary order, first in quantum mechanics (see Chapter 5 in [6]), later in quantum field theory in 14]. See also here http://klnrt.de/feynman.

[16] H. Kleinert, Converting Divergent Weak-Coupling into Exponentially Fast Convergent Strong-Coupling Expansions, Lecture presented at the 2009 meeting in Luminy, France, on Approximation and extrapolation of convergent and divergent sequences and series (http://www.ejtp.com/articles/ejtpv8i25p15.pdf).

[17] F.J. Wegner, Z. Physik B 78, 33 (1990).

[18] C.M. Bender, K.A. Milton, M. Moshe, S.S. Pinsky, and L. M. Simmons, Phys. Rev. Lett. 58, 2615 (1987); Phys. Rev. D 37, 1472 (1988); C.M. Bender and H.F. Jones, J. Math. Phys. 29, 2659 (1988); Phys. Rev. D 38, 2526 (1988).

[19] J.A. Lipa, J.A. Nissen, D. A. Stricker, D.R. Swanson, and T.C.P. Chui, Phys. Rev. B 68, 174518 (2003); M. Barmatz, I. Hahn J.A. Lipa, R.V. Duncan, Rev. Mod. Phys. 79, 1 (2007). Also see picture on the title-page of the textbook 14].

[20] S.T. Belyaev, Sov. Phys. JETP 7289 (1958). For details see Chapter 5 in the textbook A.A. Abrikosov, L.P. Gorkov, and I.E. Dzyaloshinski, Methods of Quantum Field Theory in Statistical Physics, Dover, New York, 1961. See also the early applications in quantum field theory by P.T. Mathews, A. Salam, Nuovo Cimento 12, 563 (1954), 2, 120 (1955), and later in the study of the large$N$ limit of various model field theories, such as GrossNeveu and nonlinear $\sigma$ models.

[21] See for instance H. Kleinert, Mod. Phys. Lett. B, 17, 1011 (2003) (http://klnrt.de/320).

[22] See Section 10.8 in the textbook 14], viz. Eq. (10.151). See also Eq. (1.28), expand there $f(r / \xi)=1+c(r / \xi)^{\omega}+$ $\ldots$... and compare with (96).

[23] H. Kleinert, EPL 100, 10001 (2012).

[24] S. Giorgini, L. P. Pitaevskii, and S. Stringari, Phys. Rev. A 54, R4633 (1996).

[25] F. Dalfovo, S. Giorgini, P. Pitaevskii, and S. Stringari, Rev. Mod. Phys. 71, 463512 (1999) (Fig. 3).

[26] L.V. Hau, B.D. Busch, C. Liu, Z. Dutton, M.M. Burns and J.A. Golovchenko, Phys. Rev. A 58, R54 (1998) (Fig. $2)$.

[27] F. Dalvfovo and S. Stringari, Phys. Rev. A 53, 2477 (1996).

[28] J. Tempere, F. Brosens, L. F. Lemmens, and J. T. Devreese, Phys. Rev. A 61, 043605 (2000). 